\title{
Homoclinic classes and finitude of attractors for vector fields on $n$-manifolds
}

\author{
C. M. Carballo and C. A. Morales*
}

March 6, 2001

\begin{abstract}
A homoclinic class of a vector field is the closure of the transverse homoclinic orbits associated to a hyperbolic periodic orbit. An attractor (a repeller) is a transitive set to which converges every positive (negative) nearby orbit. We show that a generic $C^{1}$ vector field on a closed $n$-manifold has either infinitely many homoclinic classes or a finite collection of attractors (repellers) whose basins form an open-dense set. This result gives an approach to a conjecture by Palis. We also prove the existence of a locally residual subset of $C^{1}$ vector fields on a 5-manifold having finitely many attractors and repellers but infinitely many homoclinic classes.
\end{abstract}

\section{Introduction}

In this paper we prove that a generic $C^{1}$ vector field on a closed $n$-manifold has either infinitely many homoclinic classes or a finite collection of attractors (repellers) whose basins form an open-dense set. This result gives an approach to a conjecture by Palis in the $C^{1}$ interior of the set of vector fields having finitely many homoclinic classes. We also prove the existence of a locally residual subset of $C^{1}$ vector fields on a 5-manifold having finitely many attractors and repellers but infinitely many homoclinic classes. This proves that the finitude of attractors and repellers does not (not even generically) imply the existence of a (finite) spectral decomposition formed by transitive sets.

Before stating our results precisely, we introduce some definitions and present the motivations for this work. The set of $C^{1}$ vector fields on a closed manifold $M$ endowed with the $C^{1}$ topology is a Baire topological space that we denote

${ }^{*}$ The first author was supported by CNPq. The second author was supported by FAPERJ, CNPq, and PRONEX/Dyn. Sys. 
$\mathcal{X}^{1}(M)$. The flow generated by $X \in \mathcal{X}^{1}(M)$ is denoted by $X^{t}$. A subset of a topological space is residual if it includes a set that is a countable intersection of open-dense sets. Given an open set $\mathcal{U}$ of $\mathcal{X}^{1}(M)$ and a property $P$, we say that $a$ generic vector field in $\mathcal{U}$ satisfies $P$ if there is a residual subset $\mathcal{R}$ of $\mathcal{U}$ such that every element of $\mathcal{R}$ satisfies $P$.

An invariant set of $X \in \mathcal{X}^{1}(M)$ is transitive if it is the $\omega$-limit set of one of its orbits. Recall that the $\omega$-limit set $\omega(x)$ of a point $x$ in $M$ is the set of all accumulation points of the positive orbit of $x$. Similarly, the $\alpha$-limit set $\alpha(x)$ of $x$ is the set of all accumulation points of the negative orbit of $x$. The nonwandering set of $X$, denoted by $\Omega(X)$, is the set of $x \in M$ such that for every neighborhood $U$ of $x$ and $T>0$ there is $t>T$ such that $X^{t}(U) \cap U \neq \emptyset$.

An attractor of $X$ is a transitive set $\Lambda$ that has a neighborhood $U$ such that $X^{t}(U) \subseteq U$, for $t>0$, and $\cap_{t \geq 0} X^{t}(U)=\Lambda$. A repeller is an attractor for the time-reversed vector field $-X$. The basin of an attractor $\Lambda$ is the set of all points $x \in M$ such that $\omega(x) \subseteq \Lambda$. Similarly, the basin of a repeller $\Lambda$ is the set of all points $x \in M$ such that $\alpha(x) \subseteq \Lambda$. If $\Lambda$ is an attractor or a repeller we denote its basin by $B(\Lambda)$.

A periodic orbit of $X$ is hyperbolic if its associated Poincare map has no eigenvalues with modulus one. Similarly, a singularity $\sigma$ of $X$ is hyperbolic if the derivative $D X(\sigma)$ has no pure imaginary eigenvalues. A sink is an attracting hyperbolic closed orbit and a source is a sink for $-X$.

Given a hyperbolic periodic orbit $O$ of a vector field $X$, the sets

$$
W^{s}(O)=\left\{x \in M: X^{t}(x) \rightarrow O, \text { as } t \rightarrow \infty\right\}
$$

and

$$
W^{u}(O)=\left\{x \in M: X^{t}(x) \rightarrow O, \text { as } t \rightarrow-\infty\right\}
$$

are $C^{1}$ immersed submanifolds of $M$. A homoclinic class of $X$ is the closure of the points of transverse intersection between $W^{s}(O)$ and $W^{u}(O)$, for some hyperbolic periodic orbit $O$ of $X$. Homoclinic classes are transitive sets [9]. Similar definitions and facts hold for diffeomorphisms.

It follows from the definition of homoclinic class that both attracting and repelling hyperbolic periodic orbits are homoclinic classes. So, the finitude of homoclinic classes implies the finitude of sinks and sources. Later on we will see (Theorem A) that the finitude of homoclinic classes generically implies the finitude of attractors as well.

A central motivation for this work is the following theorem by Mañé: a generic $C^{1}$ surface diffeomorphism either has infinitely many sinks or sources or it is Axiom A without cycles [6]. In particular, if a generic $C^{1}$ diffeomorphism has only finitely many sinks and sources, then it has a finite collections of attractors 
whose basins form an open-dense set and a finite collection of repellers whose basins form an open-dense set. A recent related result [7] implies that the same conclusion is valid for $C^{1}$ vector fields on 3 -manifolds. Our first result gives a (generically) sufficient condition for the finitude of attractors for vector fields on higher dimensional manifolds. From now on, let $M$ be a closed $n$-manifold, $n \geq 3$.

Theorem A. A generic vector field in $\mathcal{X}^{1}(M)$ has either infinitely many homoclinic classes or else a finite collection of attractors whose basins form an opendense set and a finite collection of repellers whose basins form an open-dense set.

As a consequence of this, we have the following corollary dealing with the global conjecture on the finitude of attractors and their metric stability by Palis [10. Let $\mathcal{H C}^{1}(M)$ be the $C^{1}$ interior of the set of $X \in \mathcal{X}^{1}(M)$ such that $X$ has finitely many homoclinic classes.

Corollary B. If $X \in \mathcal{H C}^{1}(M)$ and $\Lambda$ is an attractor, then there is a negihborhood $\mathcal{U} \subseteq \mathcal{H C}^{1}(M)$ of $X$ such that a generic vector field in $\mathcal{U}$ (i.e. a generic perturbation of $X$ ) has a finite collection of attractors whose basins contain an open-dense subset of $B(\Lambda)$. A generic vector field in $\mathcal{H C}^{1}(M)$ has a finite collection of attractors whose basins form an open-dense set.

The result in [7] mentioned above shows that if a generic $C^{1}$ vector field on a closed 3-manifold has finitely many attractors and repellers, then it also has finitely many homoclinic classes. This is not true in higher dimensions by the following example.

Theorem C. There are a closed 5-manifold $M$ and an open subset $\mathcal{U}$ of $\mathcal{X}^{1}(M)$ such that a generic vector field in $\mathcal{U}$ has two attractors (whose basins form an open-dense set), a unique repeller (with open-dense basin), and infinitely many homoclinic classes.

We remark that both Theorem A and Corollary B hold for diffeomorphisms. Theorem $\square$ also holds for diffeomorphisms on $n$-manifolds, $n \geq 4$, as we will see later (Proposition 3.1).

\section{Proof of Theorem $\AA$ and Corollary $B$}

We start by summarizing the generic properties of $C^{1}$ vector fields that we will use and we establish a preliminary lemma that gives a sufficient condition for an invariant set to be an attracting set.

The union of the closed orbits of a vector field $X$ is denoted by $\operatorname{Crit}(X)$. Pugh's General Density Theorem [13] defines a residual subset $\mathcal{P}$ of $C^{1}$ vector 
fields which have the following properties. If $X \in \mathcal{P}$, then all its closed orbits are hyperbolic, the stable and unstable manifolds of the closed orbits intersect transversely, and $\Omega(X)=\mathrm{Cl}(\operatorname{Crit}(X))$. In particular, $X$ has a finite number of singularities.

We denote by $\mathcal{Q}$ the residual subset of $C^{1}$ vector fields introduced in [4]. If $X \in \mathcal{Q}$, then its homoclinic classes either coincide or are disjoint.

A compact set $\Lambda$ of a $C^{1}$ vector field $X$ is isolated if it has a neighborhood $U$ (called isolating block) such that $\cap_{t \in \mathbb{R}} X^{t}(U)=\Lambda$. An attracting set is an isolated set having an isolating block $U$ such that $X^{t}(U) \subset U$ for every $t>0$. A compact set $\Lambda$ is Lyapunov stable for $X$ if for every neighborhood $U$ of $\Lambda$ there is another neighborhood $V \subseteq U$ such that $X^{t}(V) \subset U$ for every $t>0$.

Given a vector field $X$ on a closed $n$-manifold $M$ we denote by $R_{X}$ the set of all $x \in M$ such that $\omega(x)$ is Lyapunov stable for $X$. We will use the following result.

2.1. Theorem. ([8]) The set

$$
\mathcal{S}=\left\{X \in \mathcal{X}^{1}(M): R_{X} \text { is residual in } M\right\}
$$

is residual in $\mathcal{X}^{1}(M)$.

The attractors of a vector field are transitive isolated Lyapunov stable sets. The lemma below implies the converse. It seems to be well known and we prove it here for completeness.

2.2. Lemma. Isolated Lyapunov stable sets are attracting sets.

Proof. Let $\Lambda$ be an isolated Lyapunov stable set of a vector field $X$. We first observe that there is an open set sequence $U_{n}$ such that $U_{n+1} \subseteq U_{n}, X^{t}\left(U_{n}\right) \subset U_{n}$, for all $n$, and $\Lambda=\cap_{n \in \mathbb{N}} U_{n}$. To see this, let $B_{n}$ be the ball of radius $1 / n$ around $\Lambda$, i.e. $B_{n}=\{x \in M: d(x, \Lambda)<1 / n\}$. By the Lyapunov stability of $\Lambda$, for each $n$, there is an open neighborhood $V_{n} \subseteq B_{n}$ of $\Lambda$ such that $X^{t}\left(V_{n}\right) \subseteq B_{n}$ for $t \geq 0$. Setting $U_{n}=\cap_{t \geq 0} X^{t}\left(V_{n}\right)$ we are done.

Now, let $U$ be an isolating block for $\Lambda$ and $n$ be such that $U_{n} \subseteq U$. Then, $X^{t}\left(U_{n}\right) \subset X^{t}(U)$ for every $t \in \mathbb{R}$. So,

$$
\cap_{t \in \mathbb{R}} X^{t}\left(U_{n}\right) \subseteq \Lambda
$$

since $U$ is an isolating block of $\Lambda$.

On the other hand, as $U_{n}$ is forward invariant, we have that $U_{n} \subseteq X^{t}\left(U_{n}\right)$ for $t \leq 0$. Then, $U_{n} \subset \cap_{t \leq 0} X^{t}\left(U_{n}\right)$ and so

$$
\cap_{t \geq 0} X^{t}\left(U_{n}\right)=U_{n} \cap\left(\cap_{t>0} X^{t}\left(U_{n}\right)\right) \subset\left(\cap_{t \leq 0} X^{t}\left(U_{n}\right)\right) \cap\left(\cap_{t>0} X^{t}\left(U_{n}\right)\right)=\cap_{t \in \mathbb{R}} X^{t}\left(U_{n}\right) .
$$




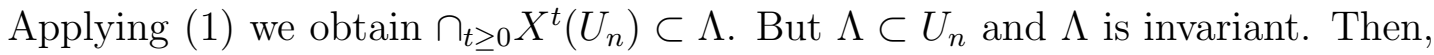
$\Lambda \subset X^{t}\left(U_{n}\right)$ for every $t \geq 0$ and so $\Lambda \subset \cap_{t \geq 0} X^{t}\left(U_{n}\right)$. This proves $\cap_{t \geq 0} X^{t}\left(U_{n}\right)=\Lambda$. Recalling that $U_{n}$ is a neighborhood of $\Lambda$, we see that $\Lambda$ is an attracting set since it is compact. This proves the lemma.

Proof of Theorem A: We define a residual subset $\mathcal{R}$ of $\mathcal{X}^{1}(M)$ by $\mathcal{R}=$ $\mathcal{P} \cap \mathcal{Q} \cap \mathcal{S}$ and consider $X \in \mathcal{R}$ so that our hypothesis implies that $X$ satisfies all the generic properties above.

If $X$ has infinitely many homoclinic classes, then we are done. If not, let $H_{1}, \ldots, H_{m}$ be the collection of all homoclinic classes of $X$. By hypothesis, Pugh's General Density Theorem applies to $X$; hence $X$ has finitely many singularities and $\Omega(X)=\mathrm{Cl}(\operatorname{Crit}(X))$. Let $\operatorname{Sing}(X)$ be the set of singularities of $X$ and $\operatorname{Per}(X)$ be the set of periodic points of $X$. Then, we have that $\Omega(X)=\operatorname{Sing}(X) \cup$ $\mathrm{Cl}(\operatorname{Per}(X))$. On the other hand, by the definition of homoclinic classes, it is clear that $\mathrm{Cl}(\operatorname{Per}(X)) \subseteq \cup_{i=1}^{m} H_{i}$ (the closure of the periodic points is contained in the closure of the union of the collection of homoclinic classes). From this, $\Omega(X) \subseteq \operatorname{Sing}(X) \cup\left(\cup_{i=1}^{m} H_{i}\right)$. Observe that we also have the other inclusion because each homoclinic class is included in the nonwandering set of $X$.

Then,

$$
\Omega(X)=\operatorname{Sing}(X) \cup\left(\cup_{i=1}^{m} H_{i}\right) .
$$

Let $\sigma_{1}, \ldots, \sigma_{l}$ be the singularities that are not contained in any homoclinic class; we have that

$$
\Omega(X)=\left\{\sigma_{1}\right\} \cup \cdots \cup\left\{\sigma_{l}\right\} \cup H_{1} \cup \cdots \cup H_{m} .
$$

Observe that, by hypothesis, $X$ satisfies the generic properties in [4]. In particular, the collection $\left\{H_{i}: i=1, \cdots, m\right\}$ is disjoint. The last equality and the fact that $\left\{H_{i}: i=1, \cdots, m\right\}$ is disjoint imply that every $H_{i}$ is $\Omega$-isolated, i.e. $\Omega(X) \backslash H_{i}$ is a closed set. By $\|$ w we conclude that every $H_{i}$ is an isolated set.

Let $\Lambda$ be an attractor of $X$ and $x \in M$ such that its $\omega$-limit set $\omega(x)=\Lambda$. In particular, $\omega(x)$ is Lyapunov stable. We claim that $\Lambda$ is one of the sets in the decomposition. Indeed, we have the following:

Claim. Let $X$ as above and $x \in M$. If $\omega(x)$ is Lyapunov stable, then $\omega(x)$ is either one of the $\sigma_{i}$ 's or one of the $H_{i}$ 's.

Proof. Clearly $\omega(x) \subset \Omega(X)$. By assumption we have $\Omega(X)=\left\{\sigma_{1}\right\} \cup \cdots \cup\left\{\sigma_{l}\right\} \cup$ $H_{1} \cup \cdots \cup H_{m}$ (disjoint union). Suppose that $\omega(x) \cap H_{i} \neq \emptyset$ for some $i$. Then, $\omega(x) \subseteq H_{i}$ since $\omega(x) \subseteq \Omega(x)$ is connected and the above union is disjoint. As $\omega(x)$ is Lyapunov stable and $H_{i}$ is transitive, we conclude that $H_{i} \subseteq \omega(x)$. We conclude that $\omega(x)=H_{i}$. 
Next suppose that $\omega(x) \cap\left\{\sigma_{i}\right\} \neq \emptyset$ for some $i$. Then, $\omega(x) \subseteq\left\{\sigma_{i}\right\}$ by connectedness once more. We conclude that $\omega(x)=\left\{\sigma_{i}\right\}$. To finish we note that $\omega(x)$ must intersect either $\left\{\sigma_{i}\right\}$ or $H_{i}$ (for some $i$ ) since $\omega(x) \subseteq \Omega(X)$ and $\Omega(X)=\left\{\sigma_{1}\right\} \cup \cdots \cup\left\{\sigma_{l}\right\} \cup H_{1} \cup \cdots \cup H_{m}$. This proves the claim.

The Claim implies that $X$ has only finitely many attractors (because any of them is either $\sigma_{i}$ or $H_{i}$ for some $i$ ). Let us prove that the union of the basins of the attractors of $X$ is an open-dense set of $M$. By hypothesis $X \in \mathcal{S}$. So, by Theorem 2.1, the set $R_{X}$ of $x \in M$ such that $\omega(x)$ is Lyapunov stable is residual in $M$. Denote $B(X)=\cup\{B(\Lambda): \Lambda$ is an attractor of $X\}$. We will prove that $R_{X} \subseteq B(X)$. Indeed, choose $x \in R_{X}$. Then, $\omega(x)$ is Lyapunov stable. The Claim implies that $\omega(x)$ is either $\sigma_{i}$ or $H_{i}$ for some $i$. If $\omega(x)=\left\{\sigma_{i}\right\}$ for some $i$, then we have that $\sigma_{i}$ is a sink since $\sigma_{i}$ is hyperbolic and $\omega(x)$ is Lyapunov stable. In particular, $\omega(x)$ is an attractor of $X$. If $\omega(x)=H_{i}$ for some $i$ we have that $H_{i}$ is Lyapunov stable. As $H_{i}$ is isolated we can apply Lemma 2.2 to obtain that $H_{i}$ is an attracting set. As $H_{i}$ is transitive we conclude that $H_{i}$ (and hence $\omega(x)$ ) is an attractor. So, $\omega(x)$ is an attractor in this case as well. This proves that $R_{X} \subseteq B(X)$. As $R_{X}$ is residual we conclude that $B(X)$ is dense. As $B(X)$ is obviously open we conclude that $B(X)$ is an open-dense set of $M$.

We have proved that a generic $X \in \mathcal{X}^{1}(M)$ has either infinitely many homoclinic classes or a finite collection of attractors with open-dense basin. The same argument applied to the time-reversed vector field $-X$ shows that a generic $X \in \mathcal{X}^{1}(M)$ satisfies a similar conclusion for repellers (instead of attractors). The proof follows since the intersection of residual sets is residual as well.

Proof of Corollary B: The second part of Corollary B follows directly from Theorem $\mathrm{A}$. To prove the first part, we let $X \in \mathcal{H C}^{1}(M)$ and $\Lambda$ be an attractor of $X$. Let $Y$ be a generic $C^{1}$ perturbation of $X$ so that we can assume that $Y \in \mathcal{R} \cap \mathcal{H C}^{1}(M)$. By Theorem $\mathrm{A}$ we have that $Y$ has finitely many attractors whose basins of attraction form an open-dense set of $M$. As before, we let $B(Y)$ denote the union of the basins of the attractors of $Y$. Now, let $\Lambda_{1}, \ldots, \Lambda_{k}$ be the attractors of $Y$ whose basins meet the basin of $\Lambda$; i.e. such that $B\left(\Lambda_{i}\right) \cap B(\Lambda) \neq \emptyset$, for $i=1, \ldots, k$. We must show that $\left(\cup_{i=1}^{k} B\left(\Lambda_{i}\right)\right) \cap B(\Lambda)$ is an open-dense subset of $B(\Lambda)$. It is clear that it is open. It is also clear that it is dense because $\left(\cup_{i=1}^{k} B\left(\Lambda_{i}\right)\right) \cap B(\Lambda) \supseteq B(Y) \cap B(\Lambda)$ and we know that $B(Y)$ is dense in $M$. This proves the result.

\section{Proof of Theorem $\mathrm{C}$}

In this section we prove Theorem C. The proof is a simple application of the normally hyperbolic theory and [3]. The vector field (and the manifold) in the 
proposition will be obtained by suspending a suitable $C^{\infty}$ diffeomorphism $F$ in the 4-sphere $S^{4}$ exhibiting a normally expanding submanifold diffeomorphic to the 3 -sphere $S^{3}$. In such submanifold $F$ has two saddle-type fixed points (with complex eigenvalues) persistently connected in the sense of [3]. Appart from this, $F$ has two sinks attracting every positive orbit outside $S^{3}$. It would be interesting to prove the existence of a closed manifold of dimension $n \leq 4$ satisfying the conclusion of Theorem $\mathbf{Q}$ (see also [1]).

Throughout, $M$ is a closed manifold and $\operatorname{Diff}^{r}(M)$ is the space of $C^{r}$ diffeomorphisms of $M, r \geq 1$. The proof of Theorem $\mathbb{Q}$ follows by suspending the following example.

3.1. Proposition. There are $F \in \operatorname{Diff}^{1}\left(S^{4}\right)$, a neighborhood $\mathcal{U} \subseteq \operatorname{Diff}^{1}\left(S^{4}\right)$ of $F$ and a residual subset $\mathcal{R}$ of $\mathcal{U}$ such that:

1. if $G \in \mathcal{U}$, then $G$ has two attractors (whose basin form an open-dense set) and a repeller (with open-dense basin), and

2. if $G \in \mathcal{R}$, then $G$ has infinitely many homoclinic classes.

To prove this proposition we need to recall some concepts of normally hyperbolic theory. Given $F \in \operatorname{Diff}^{1}(M)$ and a closed invariant submanifold $N \subset M$ of $F$, we say that $N$ is normally expanding for $F$ if there are constants $\sigma>1$ and a continuous invariant splitting $T M=E_{N}^{u} \oplus T N$ over $N$ such that

$$
\left\|D F^{-n}(x) / E_{x}^{u}\right\| \leq \sigma^{-n},
$$

and

$$
\left\|D F^{-n}(x) / E_{x}^{u}\right\| \cdot\left\|D F^{n}\left(F^{-n}(x)\right) / T N_{F^{-n}(x)}\right\| \leq \sigma^{-n}
$$

for every $n \in \mathbb{N}$ and $x \in N$.

3.2. Lemma. Let $M$ be a closed manifold, $F \in \operatorname{Diff}^{1}(M)$, and $N$ be a normally expanding manifold of $F$. Suppose that there is a neighborhood $\mathcal{U} \subseteq \operatorname{Diff}^{1}(N)$ of $F / N$ and a residual subset $\mathcal{R}$ of $\mathcal{U}$ such that:

1. if $g \in \mathcal{U}$, then $g$ has a unique repeller, and

2. if $g \in \mathcal{R}$, then $g$ has infinitely many sinks.

Then, there is a neighborhood $\mathcal{U}^{\prime} \subseteq \operatorname{Diff}^{1}(M)$ of $F$ and a residual subset $\mathcal{R}^{\prime}$ of $\mathcal{U}^{\prime}$ such that:

- if $G \in \mathcal{U}^{\prime}$, then $G$ has a unique repeller close to $N$, and

- if $G \in \mathcal{R}^{\prime}$, then $G$ has infinitely many homoclinic classes. 
Proof. Note that $N$ is a normally hyperbolic manifold of $F$ (see [12]). It follows that there is a neighborhood $\mathcal{U}_{0}^{\prime}$ of $F$ where the normally expanding manifold $N$ has a continuation $N_{G}$, for every $G \in \mathcal{U}_{0}^{\prime}$. Moreover, for every $G$ which is $C^{1}$ close to $F$ there is a $C^{1}$ diffeomorphism $h_{G}: N_{G} \rightarrow N$. $h_{G}$ varies continuously with $G$ and $h_{F}: N \rightarrow N$ is the identity map.

Using $h_{G}$ we define $g_{G} \in \operatorname{Diff}^{1}(N)$ by

$$
g_{G}=h_{G} \circ\left(G / N_{G}\right) \circ h_{G}^{-1} .
$$

The map $\Phi: G \mapsto g_{G}$ is continuous in $\mathcal{U}_{0}^{\prime}$ (see the remark in Appendix 1 of [12]) and $\Phi(F)=F / N$.

As $\mathcal{R}$ is residual in $\mathcal{U}$ we have that $\mathcal{R}=\cup_{n} \mathcal{U}_{n}$ for some open-dense set sequence $\mathcal{U}_{n}$ of $\mathcal{U}$. Define $\mathcal{U}^{\prime}=\Phi^{-1}(\mathcal{U})$ and $\mathcal{U}_{n}^{\prime}=\Phi^{-1}\left(\mathcal{U}_{n}\right)$ for all $n$. Clearly both $\mathcal{U}^{\prime}$ and $\mathcal{U}_{n}^{\prime}$ are open sets, and $\mathcal{U}_{n}^{\prime} \subseteq \mathcal{U}^{\prime}$. It is also clear that every $G \in \mathcal{U}^{\prime}$ has a unique repeller close to $N$.

We claim that $\mathcal{U}_{n}^{\prime}$ is dense in $\mathcal{U}^{\prime}$ for every $n$. Indeed, fix $n \in \mathbb{N}$ and $G \in \mathcal{U}^{\prime}$. Thus, $\Phi(G)=g_{G} \in \mathcal{U}$. The denseness of $\mathcal{U}_{n}$ in $\mathcal{U}$ allows us to choose a sequence $g_{k} \in \mathcal{U}_{n}$ such that $g_{k} \rightarrow g_{G}$, as $k \rightarrow \infty$. Define $\widehat{g}_{k}: N_{G} \rightarrow N_{G}$ by

$$
\widehat{g}_{k}=h_{G}^{-1} \circ g_{k} \circ h_{G} .
$$

It follows that $\widehat{g}_{k} \rightarrow G / N_{G}$, as $k \rightarrow \infty$. Then, for every $k$ large there is an extension $G_{k} \in \operatorname{Diff}^{1}(M)$ of $\widehat{g}_{k}$ to $M$ so that the sequence $G_{k}$ converges to $G$ (see, for instance, [11, p. 8] or the proof of Theorem 3 in [5, p. 107]). Indeed, there is a sequence $G_{k} \in \operatorname{Diff}^{1}(M)$ such that $G_{k} \rightarrow G$, as $k \rightarrow \infty, G_{k}\left(N_{G}\right)=N_{G}$, and $G_{k} / N_{G}=\widehat{g}_{k}$ for all $k$ large. In particular, $G_{k} \in \mathcal{U}^{\prime}$. The uniqueness of the continuation of $N$ for $G$ close to $F$ then implies that $h_{G_{k}}=h_{G}, N_{G_{k}}=N_{G}$, and $\Phi\left(G_{k}\right)=h_{G} \circ\left(G_{k} / N_{G}\right) \circ h_{G}^{-1}=g_{k} \in \mathcal{U}_{n}^{\prime}$. This proves that $G_{k} \in \mathcal{U}_{n}^{\prime}$. The claim follows since $G_{k} \rightarrow G$, as $k \rightarrow \infty$.

By the claim we have that $\mathcal{R}^{\prime}=\cap_{n} \mathcal{U}_{n}^{\prime}$ is residual in $\mathcal{U}^{\prime}$. Observe that if $G \in \mathcal{R}^{\prime}$, then $\Phi(G) \in \mathcal{U}_{n}$, for all $n$, yielding $\Phi(G) \in \mathcal{R}$. We conclude that $\Phi(G)$ has infinitely many sinks. As $G / N_{G}$ and $\Phi(G)$ are conjugated we conclude that $G / N_{G}$ has infinitely many sinks too. But $N_{G}$ is normally expanding, so, the infinitely many sinks of $G / N_{G}$ correspond to infinitely many saddle-type hyperbolic periodic orbits of $G$ whose homoclinic classes are trivial. We conclude that every $G \in \mathcal{R}^{\prime}$ has infinitely many homoclinic classes and the lemma follows.

Let $N$ be a closed manifold and $f \in \operatorname{Diff}^{1}(N)$. Following [3] we say that two hyperbolic periodic points $P^{\prime}, Q^{\prime}$ of $f$ are persistently connected if there is a neighborhood $\mathcal{U} \subseteq \operatorname{Diff}^{1}(N)$ of $f$ and a dense subset $\mathcal{D}$ of $\mathcal{U}$ such that for every $g \in \mathcal{D}$ there is a transitive set of $g$ containing the continuations of both $P^{\prime}$ and $Q^{\prime}$. 
3.3. Theorem. (汭, Theorem C]) Let $N$ be a closed 3-manifold, $f \in \operatorname{Diff}^{1}(N)$ and $P^{\prime}, Q^{\prime}$ be different hyperbolic fixed points of $f$ which are persistently connected. Suppose that $P^{\prime}$ has a nonreal complex eigenvalue with modulus $<1$, that $Q^{\prime}$ has a nonreal complex eigenvalue with modulus $>1$, and that the Jacobian of $f$ at $P^{\prime}$ $i s<1$. Then there is a neighborhood $\mathcal{U}$ of $f$ and a residual subset $\mathcal{R}$ of $\mathcal{U}$ such that if $G \in \mathcal{R}$, then $G$ has infinitely many sinks.

This result is used to prove the following lemma.

3.4. Lemma. There are $f_{0} \in \operatorname{Diff}^{1}\left(S^{3}\right)$, a neighborhhod $\mathcal{U} \subseteq \operatorname{Diff}^{1}\left(S^{3}\right)$ of $f_{0}$, and a residual subset $\mathcal{R}$ of $\mathcal{U}$ such that:

1. if $g \in \mathcal{U}$, then $g$ has a unique repeller, and

2. if $g \in \mathcal{R}$, then $g$ has infinitely many sinks.

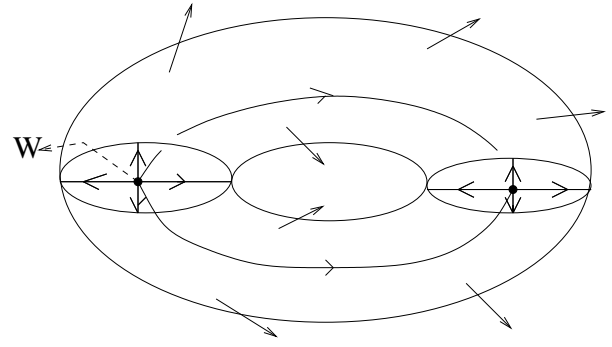

$\mathrm{ST}_{1}$

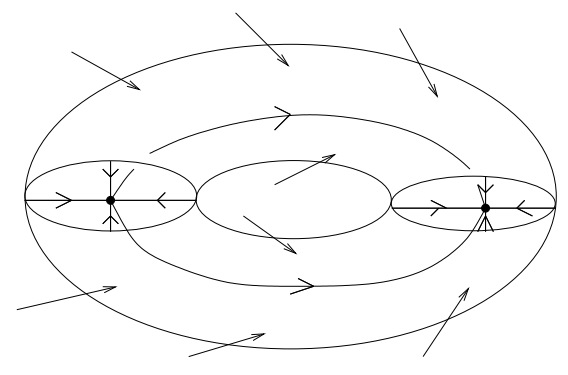

$\mathrm{ST}_{0}$

Figure 1: The map $g_{0}$.

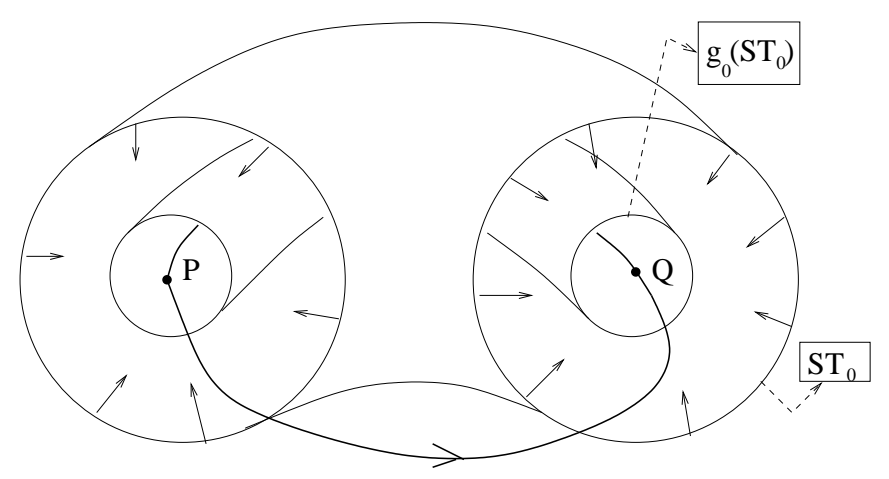

Figure 2: Configuration in $S T_{0}$. 
Proof. Consider $S^{3}$ as two solid tori $S T_{1}, S T_{0}$ glued along their boundary tori. Let $g_{0} \in \operatorname{Diff}^{\infty}\left(S^{3}\right)$ be as in Figure [1. Note that $g_{0}$ has four fixed points: two saddles, one sink, and one source, denoted by $W$ in Figure 1 . The orbits in $S T_{1}$ exit $S T_{1}$ and go to $S T_{0}$ except for the ones in the middle circle. We obtain the required diffeomorphism $f_{0}$ by modifying $g_{0}$ inside $S T_{0}$.

First, we observe that the configuration inside the solid torus $S T_{0}$ is as in Figure 2. In particular, $S T_{0}$ is contracted inside it in the way described in Figure 2. Two fixed points $P, Q$ are indicated in that figure.

We deform $g_{0}\left(S T_{0}\right)$, the image of $S T_{0}$ by $g_{0}$, to obtain $g_{1} \in \operatorname{Diff}^{1}\left(S^{3}\right)$ as in Figure . 3 . The modification produces a horseshoe in the meridian disks of $S T_{0}$ as indicated; observe that $Q$ and $P$ are fixed by $g_{1}$. The modification also produces two fixed points $Q^{\prime}, P^{\prime}$ of $g_{1}$ described in Figure 3. Note that $Q^{\prime}$ (resp. $P^{\prime}$ ) and $P$ (resp. $Q$ ) are homoclinically related. We also require the resulting map $g_{1}$ to be dissipative (Jacobian $<1)$ in $S T_{0}$.

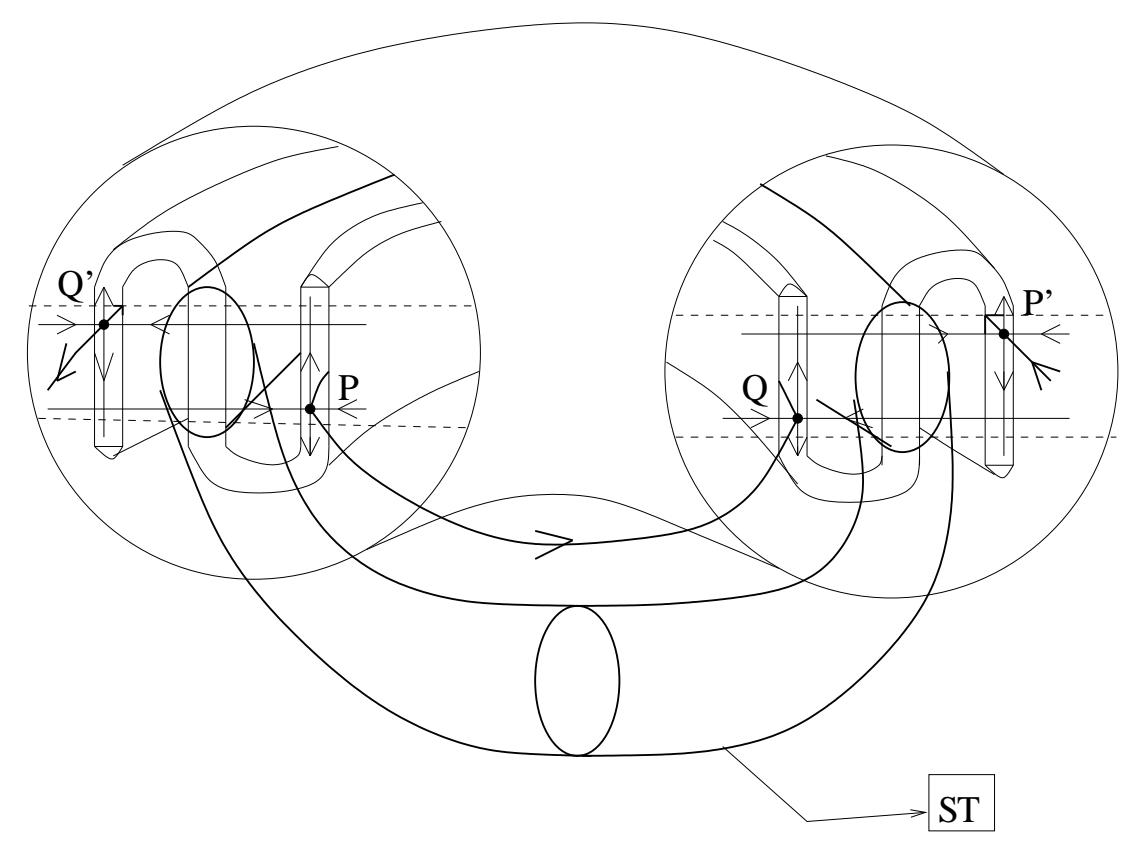

Figure 3: Creating a heterodimensional cycle.

Next, we modify $g_{1}$ to obtain $g_{2} \in \operatorname{Diff}^{1}\left(S^{3}\right)$ by performing a rotation supported on the middle solid torus $S T$ indicated in Figure 3. This rotation is done to create a heterodimensional cycle involving $P, Q$ (see [3]). We still have that the map $g_{2}$ is dissipative in $S T_{0}$.

Finally, we modify $g_{2}$ in order to obtain $g_{3} \in \operatorname{Diff}^{1}\left(S^{3}\right)$ such that the expanding eigenvalues of $Q^{\prime}$ (resp. contracting eigenvalues of $P^{\prime}$ ) become complex 
conjugated. This can be done without destroying the heterodimensional cycle involving $P, Q$.

Unfolding the cycle we create a blender ("mélangeur") containing $P, Q$ (see [2]). The existence of the blender guarantees that $P^{\prime}$ and $Q^{\prime}$ are persistently connected. This procedure is similar to the one described in [3, p. 149].

Define $f_{0}=g_{3}$. By Theorem 3.3 it follows that there is a neighborhood $\mathcal{U}$ of $f_{0}$ and $\mathcal{R}$ residual in $\mathcal{U}$ such that every $g \in \mathcal{R}$ has infinitely many sinks. We observe that every $g \in \mathcal{U}$ has a unique repeller: the continuation of the source $W \in S T_{1}$ of $g_{0}$ indicated in Figure 1 (note that $W$ is also a source of $f_{0}$ ). This is because any other repeller of $g$ close to $f_{0}$ must belong to the dissipative region $S T_{0}$ but this is impossible by the dissipative condition. This proves the result.

Proof of Proposition 3.1: Let $f_{0} \in \operatorname{Diff}^{1}\left(S^{3}\right)$ be as in the previous lemma (Lemma 3.4). Let $B$ be a closed 4-ball in $\mathbb{R}^{4}$ centered at $b$. Note that $\partial B=S^{3}$. We extend $f_{0} \in \operatorname{Diff}^{1}\left(S^{3}\right)$ to $F_{0} \in \operatorname{Diff}^{1}(B)$ such that $S^{3}$ is a normally expanding submanifold of $F_{0}$ and $b$ is a sink of $F_{0}$ attracting every forward orbit of $F_{0}$ outside $S^{3}$. Consider the double manifold $2 B=S^{4}$ and the double map $F=2 F_{0}$. It follows that $N=S^{3}$ is a normally expanding submanifold of $F$ and that $F$ has two sinks attracting all the forward orbits outside $N$. The properties (1) and (2) of Lemma 3.4 imply that $F / N=f_{0}$ satisfies the properties (1) and (2) of Lemma 3.2 for some neighborhood $\mathcal{U} \subseteq \operatorname{Diff}^{1}(N)$ and some residual subset $\mathcal{R}$ of $\mathcal{U}$. Then, by Lemma 3.2, there is a neighborhood $\mathcal{U}^{\prime} \subseteq \operatorname{Diff}^{1}\left(S^{4}\right)$ of $F$ and a residual subset $\mathcal{R}^{\prime}$ of $\mathcal{U}^{\prime}$ such that every $G \in \mathcal{U}^{\prime}$ has only one repeller close to $N$ and every $G \in \mathcal{R}^{\prime}$ has infinitely many homoclinic classes. We conclude that every $G \in \mathcal{U}^{\prime}$ has a unique repeller since any other repeller must be far from $N$, and so, it must be the continuation of one of the sinks of $F$, a contradiction. Finally we observe that every $G$ close to $F$ has two attractors: the continuation of the sinks of $F$ (whose basin form an open-dense set of $S^{4}$ ), and a unique repeller which is the continuation of the source $W$ in Figure 1 (with open-dense basin). This completes the proof.

Using the methods of this section one can prove the following result. There is an open set $\mathcal{U} \subseteq \operatorname{Diff}^{1}\left(S^{3} \times S^{1}\right)$ and a residual subset $\mathcal{R} \subseteq \mathcal{U}$ such that: (1) if $g \in \mathcal{U}$, then $g$ has an attractor and a repeller (both with open-dense basin), and (2) if $g \in \mathcal{R}$, then $g$ has infinitely many homoclinic classes. This allows us to reduce the number of attractors (repellers) to one, the minimal possible.

It is interesting to observe that the diffeomorphisms $F$ in the example of Proposition 3.1 have a continuous dominated splitting $T M_{\Omega(F)}=E^{s} \oplus E^{c} \oplus E^{u}$ such that $E^{s}$ is contracting, $E^{u}$ is expanding, and either $E^{s}$ or $E^{u}$ is nontrivial. This shows that the existence of such a splitting does not imply the existence of a (finite) spectral decomposition of the nonwandering set. 


\section{References}

[1] F. Abdenur. Generic robustness of spectral decompositions. In preparation.

[2] C. Bonatti and L. J. Díaz. Nonhyperbolic transitive diffeomorphisms. Ann. of Math., 143:357-396, 1996.

[3] C. Bonatti and L. J. Díaz. Connexions heterocliniques et genericité d'une infinité de puits ou de sources. Ann. Sci. École Norm. Sup., 32:135-150, 1999.

[4] C. M. Carballo, C. A. Morales, and M. J. Pacifico. Homoclinic classes for generic $C^{1}$ vector fields. Preprint MAT.07, PUC-Rio, 2000.

[5] E. L. Lima. Introdução à Topologia Diferencial. IMPA, 1999.

[6] R. Mañé. An ergodic closing lemma. Ann. of Math., 116:503-540, 1982.

[7] C. A. Morales and M. J. Pacifico. A dichotomy for generic three-dimensional vector fields. Preprint, 2000.

[8] C. A. Morales and M. J. Pacifico. Lyapunov stability of generic $\omega$-limit sets. Preprint, 2000.

[9] S. Newhouse. Lectures on Dynamical Systems. In Dynamical Systems, pages 1-114. Birkhäuser, Boston, 1978.

[10] J. Palis. A global view of dynamics and a conjecture on the denseness of finitude of attractors. Astérisque, 261:335-347, 2000.

[11] J. Palis and W. de Melo. Geometric Theory of Dynamical Systems. SpringerVerlag, Berlin, 1982.

[12] J. Palis and F. Takens. Topological equivalence of normally hyperbolic dynamical systems. Topology, 16:335-345, 1977.

[13] C. Pugh. An improved closing lemma and a general density theorem. Amer. J. Math., 89:1010-1021, 1967.
C. M. Carballo
C. A. Morales
PUC-Rio
Instituto de Matemática
Dto. de Matemática
Universidade Federal do Rio de Janeiro
Rua Marquês de São Vicente, 225
C. P. 68.530
22453-900, Rio de Janeiro, RJ
21945-970, Rio de Janeiro, RJ
Brazil
Brazil
carballo@mat.puc-rio.br
morales@impa.br 